\title{
Anti-tumor effect of endostatin in a sleep-apnea mouse model with tumor
}

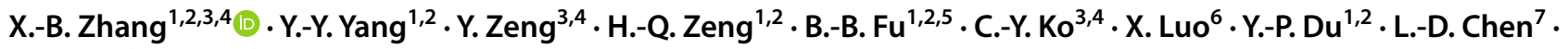 \\ Y.-T. Lai ${ }^{1,2} \cdot$ Y. Wu ${ }^{1,2}$
}

Received: 6 August 2018 / Accepted: 11 September 2018 / Published online: 6 October 2018

(c) The Author(s) 2018

\begin{abstract}
Background Obstructive sleep apnea (OSA) is associated with cancer incidence and mortality. The underlying mechanism is unclear. This study aims to evaluate the influence of intermittent hypoxia (IH), a novel hallmark of OSA, on tumor and to access the anti-tumor effect of endostatin on a mouse model with OSA.

Methods The C57BL/6 J mice were randomly classified into four groups: control (normoxia) (CTL), control plus endostatin $(\mathrm{CTL}+\mathrm{ED}), \mathrm{IH}$, and IH plus endostatin $(\mathrm{IH}+\mathrm{ED})$. Mice in IH and IH + ED groups were subjected to IH $8 \mathrm{~h}$ per day in 5 weeks. Lewis lung cancer cells were injected into the flank of each mouse after 1 week of IH exposure. Endostatin was also intraperitoneally injected after tumor volume reached about $200 \mathrm{~mm}^{3}$. The maximum standard uptake values (SUVmax) were detected by micro-positron emission tomography-computed tomography (micro-PET-CT) imaging prior and postendostatin administration. Microvessel density (MVD) and vascular endothelial growth factor (VEGF) were determined for evaluating the anti-tumor effect of endostatin among the normoxia and IH conditions.

Results Mice had higher SUVmax in the IH group than the CTL group $(p<0.01)$. When compared with mice in the CTL group, those in the IH group had significantly greater MVD values $(p<0.001)$. The SUVmax can be attenuated by endostatin both in the CTL $(p<0.01)$ and IH conditions $(p<0.001)$. When compared with CTL group, mice in the IH group had increased MVD values $(p<0.001)$ and VEGF expression both at mRNA $(p<0.05)$ and protein levels $(p<0.001$ in western blotting results). Treatment with endostatin attenuated serum and tissue VEGF levels, lowering the MVD values. As compared to normoxia condition, the endostatin-therapeutic effects were more significant under the IH condition $(p<0.05$ in western blotting results).

Conclusions Micro-PET-CT imaging is a promising non-invasive technique to evaluate the tumor metabolic characteristics under IH condition in vivo. The anti-tumor effect of endostatin under IH condition is superior to that of the normoxia condition.
\end{abstract}

Keywords Intermittent hypoxia $\cdot$ Sleep apnea $\cdot$ Tumor $\cdot$ Endostatin $\cdot$ Positron emission tomography

\section{Abbreviations list}

CTL Control

$\mathrm{CTL}+\mathrm{ED} \quad$ Control + endostatin

EGFR Epidermal growth factor receptor

HE Hematoxylin-eosin

X.-B. Zhang, Y.-Y. Yang, H.-Q. Zeng and L.-D. Chen contributed equally to this work.

\author{
Y. Zeng \\ ymzeng08@163.com \\ $\triangle$ H.-Q. Zeng \\ zhq20071212@xmu.edu.cn
}

Extended author information available on the last page of the article

$\begin{array}{ll}\text { IH } & \text { Intermittent hypoxia } \\ \text { IHC } & \text { Immunohistochemistry } \\ \text { IH +ED } & \text { Intermittent hypoxia + endostatin } \\ \text { IOD } & \text { Integrated optical density } \\ \text { MVD } & \text { Microvessel density } \\ \text { Micro-PET-CT } & \begin{array}{l}\text { Micro-positron emission tomography- } \\ \text { computer tomography }\end{array} \\ \text { OSA } & \text { Obstructive sleep apnea } \\ \text { qRT-PCR } & \text { Quantitative real-time polymerase chain } \\ & \text { reaction } \\ \text { SUVmax } & \text { Maximum standard uptake values } \\ \text { VEGF } & \text { Vascular endothelial growth factor }\end{array}$




\section{Background}

Obstructive sleep apnea (OSA) is a prevalent medical condition characterized by recurrent collapse of the upper airway during sleep. Its pathophysiological features are intermittent hypoxia (IH) and sleep fragmentation [1]. A large number of data demonstrated that OSA is associated with cardiovascular, cognitive and metabolic morbidities $[1,2]$. More recently, establishing the relationship between OSA and cancer is an active research topic in the medical community. On one side, high incidence and mortality of cancer in OSA population were detected [3-7]. On the other, researches also showed increased incidence of sleep disorder breathing among various types of cancer patients $[8,9]$. OSA can accelerate cancer progress, aggressiveness, and mortality $[3,7,10]$. As a novel pathophysiological feature of OSA, IH is closely correlated with tumor $[11,12]$. Both in vivo and in vitro studies confirmed that IH promotes various tumor growth, migration, invasiveness, and metastasis [13-18].

Although preliminary studies implicated that IH leads to tumor progression, the potential molecular mechanisms are still far from conclusion. Furthermore, little work has been done to evaluate the efficacy of the anti-tumor drug on an animal model with OSA.

Nowadays, endostatin has been administrated as an effective therapy for cancer [19, 20]. Researchers found that endostatin enhances radioresponse by normalizing tumor vasculature and relieving hypoxia [21], and reduces vascular endothelial growth factor (VEGF) levels and microvessel density (MVD) on a cancerous mouse [22]. Prior experimental studies delineated that IH enhances angiogenesis and the expression of VEGF [23, 24], which contributes to tumor growth, migration, invasiveness, and metastasis [13, 25, 26]. However, it remains unknown if the anti-tumor efficacy of endostatin under the IH condition is superior to that of the normoxia condition. Therefore, the goal of the present study was to evaluate the effect of endostatin on tumor progression in an IH mouse model mimicking OSA via micro-positron emission tomography (micro-PET) imaging.

\section{Materials and methods}

The protocol was approved by the Ethics Committee of Zhongshan Hospital, Xiamen University, and conducted in accordance with the Guide for the Care and Use of Laboratory Animals [27].

\section{Animals and subgroups}

Forty-eight 7-week-old male C57BL/6 mice were purchased from Chinese Academy of Science Laboratory Animals Center in Shanghai, China. Mice were randomly assigned to the following four groups $(n=12$ in each group): control (CTL), control plus endostatin treatment $(\mathrm{CTL}+\mathrm{ED})$, intermittent hypoxia (IH), and intermittent hypoxia plus endostatin treatment $(\mathrm{IH}+\mathrm{ED})$. With the exception of the intermittent hypoxia period, all mice were housed in standard cages with 12:12-h light-dark cycle and fed and had tap water ad libitum. Body weight $(\mathrm{g})$ of each mouse was measured every week via an electronic weighing scale.

\section{Lewis lung carcinoma cell culture and tumor induction}

Lewis lung carcinoma (LLC) cells were obtained from CoBioer Biosciences Co., Ltd. Shanghai, China, and were maintained in high-glucose DMEM and supplemented with $10 \%$ fetal bovine serum (GIBCO, USA). After 1 week of IH exposure, LLC cells were subcutaneously injected $\left(1 \times 10^{6}\right.$ LLC in $100 \mu \mathrm{l}$ PBS of each mouse) into the right flank of each mouse. Tumor volume $\left(V, \mathrm{~mm}^{3}\right)$ was calculated by measuring the tumor length $(L)$ and width $(W)$ with an electronic caliper $\left(V=W^{2} \times L / 2\right)$ every 5 days after 7 days of LLC injection.

\section{Intermittent hypoxia exposure}

Mice in the IH and IH + ED groups were subjected to IH environment. Exposure was conducted as previously described [28], with some modification. Briefly, 24 mice (IH and $\mathrm{IH}+\mathrm{ED}$ groups) were caged in a self-made plexiglass chamber with one-way valves. The IH cycle included $50 \mathrm{~s}$ of nitrogen, resting for $10 \mathrm{~s}$, oxygen for $10 \mathrm{~s}$, and compressed air for $50 \mathrm{~s}$. The oxygen concentration in the chamber fluctuated between normal $(21 \pm 1 \%)$ and nadir point $(6 \pm 1 \%)$ for every $120 \mathrm{~s}$ of one IH cycle.

\section{Drug administration}

When the tumor volume reached about $200 \mathrm{~mm}^{3}$, recombined human endostatin (Endostar, Simcere-Medgenn Bio-Pharmaceutical Co., Ltd, Shandong, China) was intraperitoneally injected $(8 \mathrm{mg} / \mathrm{kg})$ daily to those mice in the CTL + ED and $\mathrm{IH}+\mathrm{ED}$ groups. 


\section{Micro-positron emission tomography-computed tomography (micro-PET-CT) and imaging analysis}

Micro-PET-CT imaging of tumor-bearing mice (for each group $n=3$ ) was implemented by a micro-PET scanner (Inveon, Siemens) when tumor volume increased about $200 \mathrm{~mm}^{3}$ (before drug injection). Before ${ }^{18} \mathrm{~F}$-FDG injection, all mice were fasted for $12 \mathrm{~h}$ but had free access to water. Mice were intraperitoneally injected with $7.4 \mathrm{MBq}$ $(200 \mu \mathrm{Ci})$ of ${ }^{18} \mathrm{~F}-\mathrm{FDG}$ under $2 \%$ isoflurane anesthesia. Imaging commenced $60 \mathrm{~min}$ after injection. Mice were placed at the center of the field of view of the micro-PET scanner in the prone position. Data were reconstructed using a two-dimensional ordered subset expectation maximum algorithm. Imaging analysis was consistent with a previous report [29]. ASIProVMTM software (CTI Concorde Microsystems) was used to collect the regions of interests (ROIs), while a computer automatically obtains an average PET unit as the uptake value. The maximal standardized uptake value (SUVmax) was calculated as the ${ }^{18} \mathrm{~F}$-FDG uptake from the greatest intensity of the tumor image. The micro-PET-CT imaging was repeated after 15 days of endostatin injection.

\section{Tissue preparation}

After the IH exposure, mice were fasted overnight prior to terminal anesthesia. After being euthanized with $3 \%$ phenobarbital $(30 \mathrm{mg} / \mathrm{kg})$ intraperitoneal injection, mice were weighed. A total of $600 \mu \mathrm{l}$ whole blood samples were collected by a direct cardiac puncture. Tumors were excised, weighed, frozen in liquid nitrogen, stored at $-80{ }^{\circ} \mathrm{C}$ for further analysis, and fixed in buffered $10 \%$ formalin for histological examination.

\section{Serum VEGF and endothelin-1 detection}

Concentrations of VEGF $(\mathrm{pg} / \mathrm{ml})$ in serum were detected with ELISA, following the manufacturer's protocol (R\&D Systems, Minneapolis, USA).

\section{Quantity real-time polymerase chain reaction}

Total RNA was extracted from tumor tissues in each group with TRIzol reagent (Invitrogen, Carlsbad, USA). After synthesizing cDNA, quantity real-time polymerase chain reaction (qRT-PCR) was conducted in the 7500 PCR system (Applied Biosystems, Foster City, USA) in accordance with the protocol of manufacture (TaKaRa, Biotechnology, Dalian, China). The primers used in the RT-PCR were forward 5'-TGTACCTCCACCATGCCAAG-3' and reverse 3'-TCTCAATCGGACGGCAGTAG-5' for VEGF, forward 5'-GTGCTATGTTGCTCTAGACTTCG-3' and reverse
3'-ATGCCACAGGATTCCATACC-5' for $\beta$-actin which was used as internal control. The relative gene expression levels were calculated with $2^{-\mathrm{DDCt}}$.

\section{Western blotting}

Total proteins were extracted from the tumor tissues with RIPA lysis buffer (Solarbio, Beijing, China) in a glass homogenizer on ice. The protein concentrations in the supernatant of homogenate were detected by the bicinchoninic acid method using a protein assay kit (Beyotime, Beijing, China). Equal amounts of protein in each group were subjected to $10 \%$ sodium dodecyl sulfate-PAGE, and then transferred to PVDF membranes. After blocking with 5\% non-fat milk for $1 \mathrm{~h}$ at room temperature, the membranes were then incubated with the following antibodies at $4{ }^{\circ} \mathrm{C}$ overnight: mouse anti-vascular endothelial growth factor (Santa Cruz Biotechnology, USA), and mouse anti-actin (Santa Cruz Biotechnology, USA). Membranes were washed and incubated with the appropriate secondary antibody for $1 \mathrm{~h}$ at room temperature. The bands were detected with an enhanced ECL kit (Clarity $^{\mathrm{TM}}$ Western ECL Substrate, Bio-Rad).

\section{Hematoxylin-eosin staining, immunohistochemistry, and microvessel density detection}

The tumor tissue in the formalin was further embedded in paraffin and sectioned into 5- $\mu \mathrm{m}$ slices. Sections were stained with hematoxylin-eosin (HE). Immunohistochemistry (IHC) was performed using primary antibodies mentioned in western blotting. Integrated optical density (IOD) was measured using Image-Pro Plus (version 6.0; Medica Cybernetics, USA). Microvessel density (MVD) values were evaluated by immunohistochemical analysis of CD34 antibody (Santa Cruz Biotechnology, USA), and counted at a magnification of $\times 400$ using the previous method [30]. One isolated brown yellow vascular endothelial cell or cell cluster in the tumor tissue was considered as one microvessel. Vessels were counted in the three highest density areas. The mean of the MVD values represents the average number derived from high-power fields of each group.

\section{Statistical analysis}

The GraphPad Prism 5.0 (GraphPad Software, Inc., La Jolla, CA, USA) and the SPSS statistical software version 21.0 (SPSS, Inc, Chicago, IL, USA) were performed for data analysis. All values are presented as mean \pm standard deviation. Data were compared using one-way analysis of variance and post hoc test, or unpaired/paired $T$ test was also conducted for further comparison between groups, 
appropriately. A $p$ value less than 0.05 was indicated as a significant difference.

\section{Results}

\section{Body weight, tumor volume and tumor weight in each group}

At the 5th week (time of killing), mice in the IH groups gained less body weight than the CTL group $(22.05 \pm 1.13$ vs. $25.04 \pm 1.79, p<0.001$ ) (Fig. 1a). Tumor volume in mice subjected to the IH was significantly greater than that of the CTL group $(3974.50 \pm 1748.20 \mathrm{vs}$. $2268.54 \pm 1874.48, p<0.001)$. Endostatin can suppress the growth of tumor volume under IH condition $(2104.50 \pm 1372.68$ in $\mathrm{IH}+\mathrm{ED}$ group vs. $3974.50 \pm 1748.20$ in IH group, $p<0.001$ ), while this phenomenon has not been observed under normoxia condition $(1956.13 \pm 1382.08$ vs. $2268.54 \pm 1874.48, p>0.05)$ (Fig. 1b, d). The tumor weight in the IH group was greater than that of the CTL group $(5.76 \pm 1.48$ vs. $4.49 \pm 0.96$, $p<0.01$ ) (Fig. 1c, d). Meanwhile, the tumor weight was lower in the mice treated with endostatin than without it $(2.93 \pm 0.91$ vs. $4.49 \pm 0.96, p<0.01$ in normoxia condition; $3.54 \pm 1.13$ vs. $5.76 \pm 1.48, p<0.001$ in IH condition). However, there is no statistical significance in decrease levels (no-ED treatment group - ED treatment group in normoxia or IH conditions) of tumor weight between normoxia and IH conditions $(1.55 \pm 0.98$ vs. $2.23 \pm 0.85, p>0.05$ ) (Fig. 1c, d).

\section{PET-CT imaging}

The PET-CT imaging results are illustrated in Fig. 2. At IH day 15, before ED administration, four groups had the similar SUVmax $(p>0.05)$. At IH day 30 , the mean SUVmax values were significantly higher in the IH group than the CTL group $(2.0 \pm 0.19$ vs. $1.5 \pm 0.13, p<0.01)$. After ED treatment, the SUVmax values both under the normoxia (CTL + ED group) and IH conditions (IH + ED group) were decreased ( $p<0.05$ in normoxia condition, $p<0.01$ in IH condition).
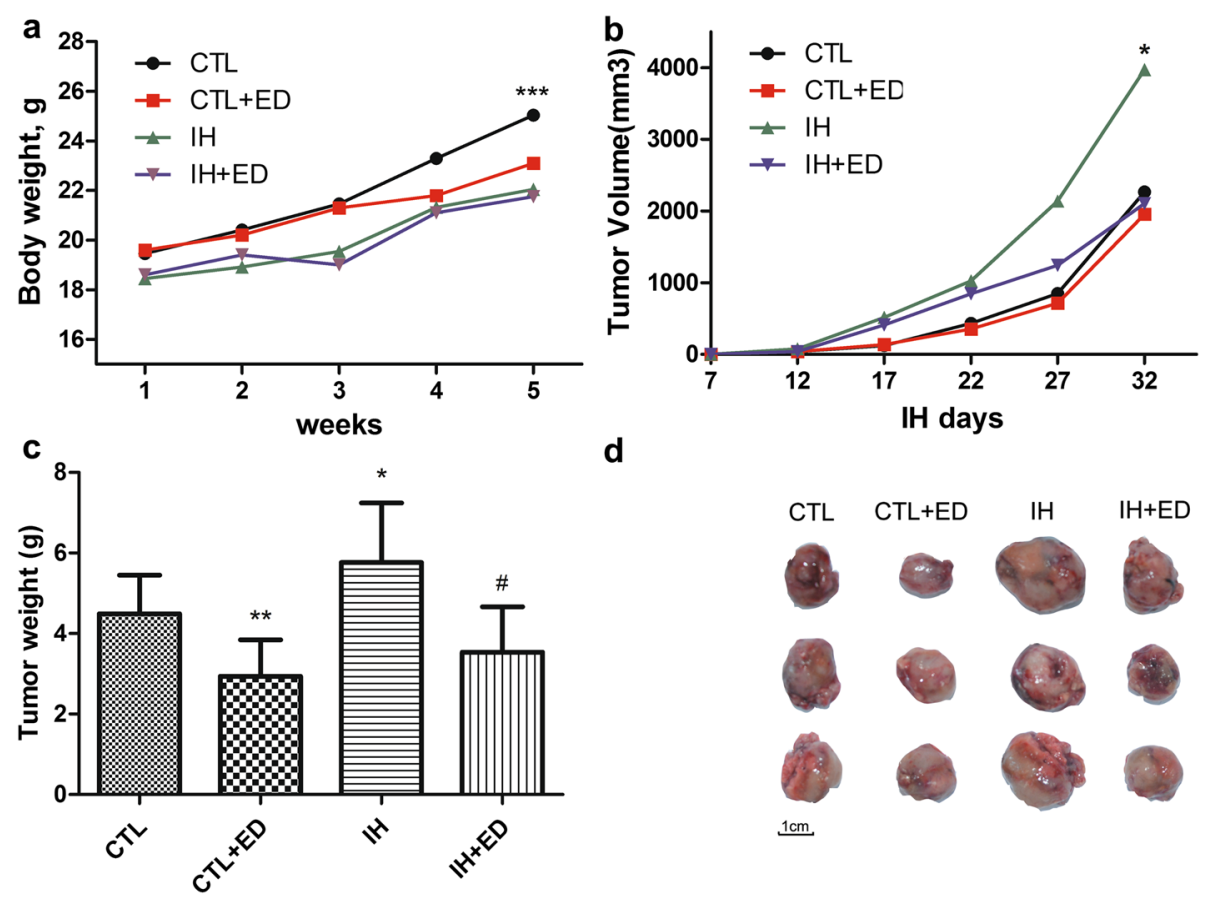

d

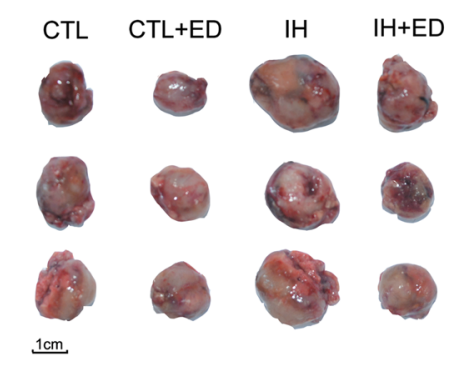

Fig. 1 Body weight, tumor volume, and tumor weight in each group. a Body weight in each group from the first week to the fifth week; mice that were exposed to the $\mathrm{IH}$ (IH and $\mathrm{IH}+\mathrm{ED}$ groups) gained less body weight than those exposed to normoxia at the fifth week $(p<0.001)$. b Tumor volume in the IH group was larger than that in the CTL group $(p<0.001)$. Endostatin treatment can lower the tumor volume under IH condition ( $p<0.001$ when IH vs. IH + ED). $\mathbf{c}$ Tumor weight in each group; tumor weight in mice subjected to the IH was heavier than that in mice under normoxia condition $(p<0.01)$; the

tumor weight in mice that received endostatin treatment was significantly lower than that in mice without endostatin treatment $(p<0.01$ in normoxia condition, $p<0.001$ in IH condition). d The tumor size in different groups. ${ }^{*} p<0.05$ when compared with CTL group; $*^{*} p<0.01$ when compared with CTL group; $* * * p<0.001$ when compared with CTL group; ${ }^{\#} p<0.05$ when compared with IH group. Abbreviations: $I H$ intermittent hypoxia; $C T L$ control group; $E D$ endostatin 
Fig. 2 Representative coronal and sagittal sections of 18F-FDG PET-CT images of LLC-bearing mice at 15-day IH (a) and 30-day IH (b). SUVmax values are presented below the images. Yellow arrows indicate tumors. Necrosis can be seen in the IH group at IH day 15 and in all groups at $\mathrm{IH}$ day 30 . $* p<0.001$ when compared with CTL group at IH day 30; ${ }^{\#} p<0.01$ when compared with CTL + ED group at IH day 15 ; ${ }^{\&} p<0.001$ when compared with $\mathrm{IH}+\mathrm{ED}$ group at IH day 15. Abbreviations: $C T L$ control group; $I H$ intermittent hypoxia; $E D$ endostatin
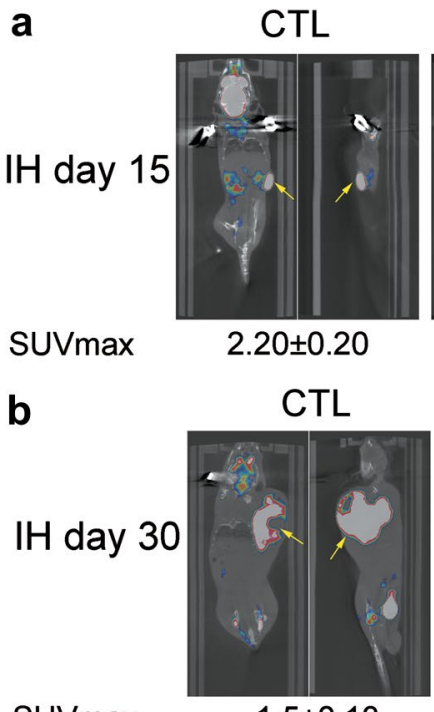

SUVmax
CTL+ED

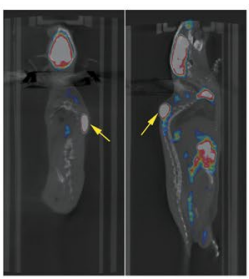

$2.30 \pm 0.41$

CTL+ED

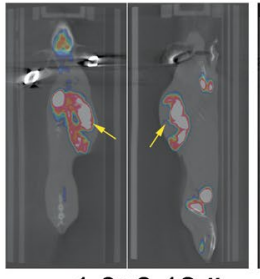

$1.3 \pm 0.12 \#$
$\mathrm{IH}$

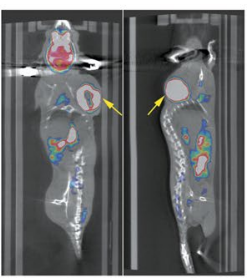

$2.1 \pm 0.34$

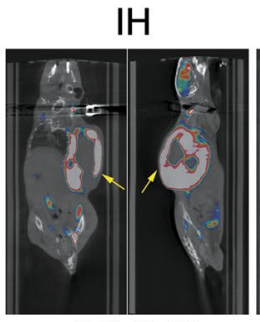

$2.0 \pm 0.19$ *
IH+ED

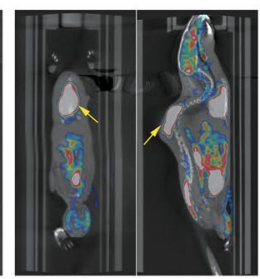

$2.5 \pm 0.41$

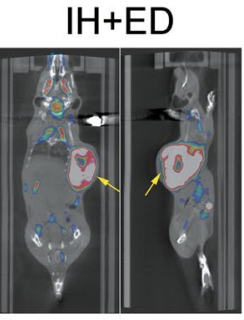

$1.4 \pm 0.20 \&$

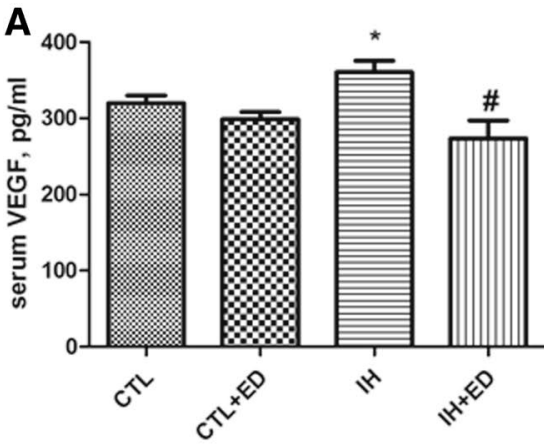

C

D
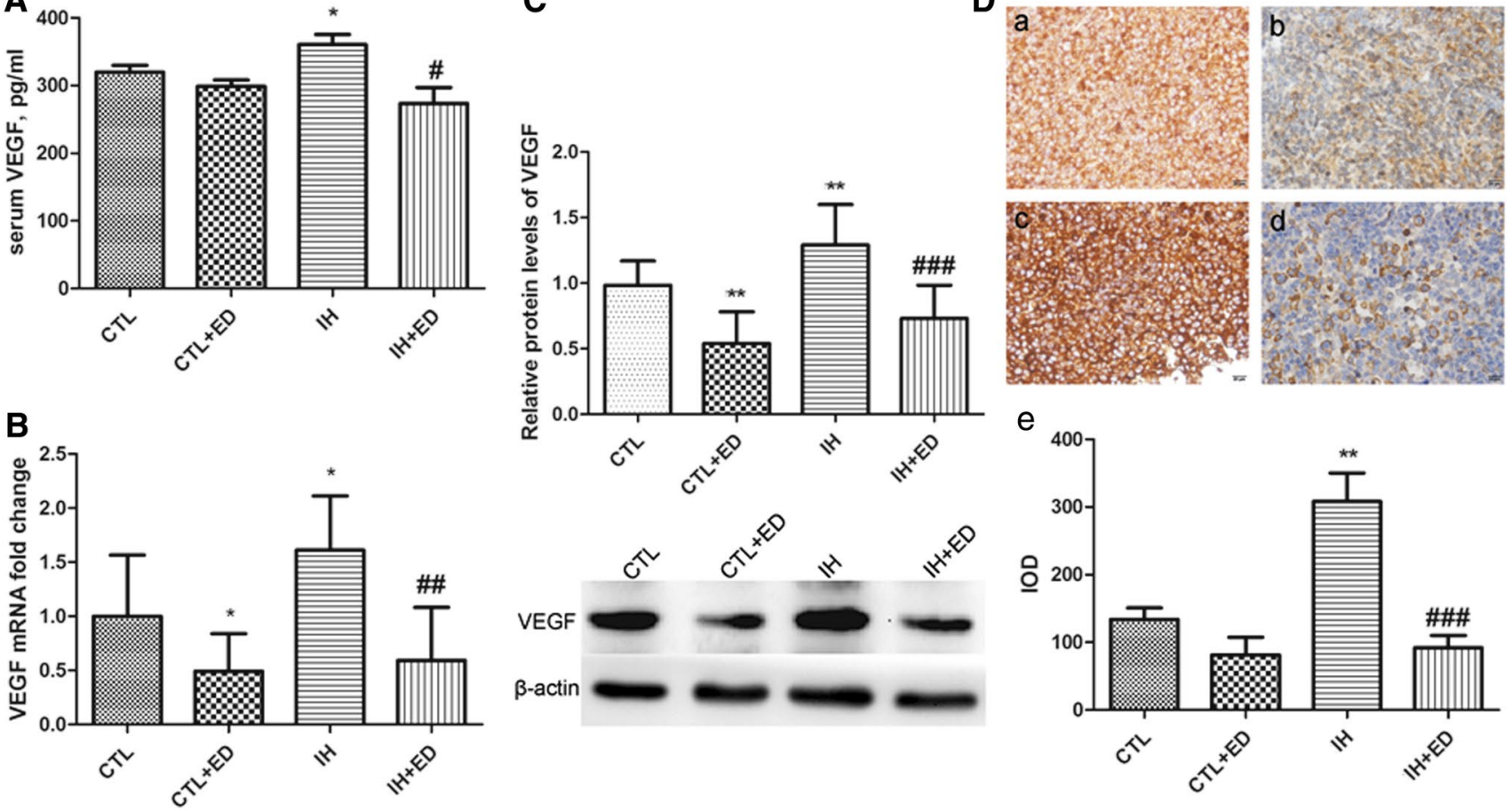

Fig. 3 Effect of endostatin on the expression of VEGF. A Serum VEGF level detection by ELISA; B RT-PCR results illustrated that compared with CTL group, mice in IH group had increased VEGF mRNA expression levels; the VEGF mRNA levels both under normoxia and IH conditions were decreased after treatment with endostatin; the degree of decline is more significant under IH condition than under normoxia condition $(p<0.05)$. C Western blotting results showed that protein levels of VEGF in IH group were greater than that in CTL group; after being treated with endostatin, reduced VEGF levels were observed both under the CTL and IH condition, the reduced levels are of greater significance in mice exposed to $\mathrm{IH}$ than those exposed to normoxia conditions. D Results of immuno- histochemical staining $(400 \times)$ by VEGF antibody demonstrated that when compared with the CTL group (a), significantly higher IOD levels were found in IH group (c); after treating with endostatin, the IOD levels were obviously declined in IH +ED group (d) $(p<0.001)$, and the declined levels in CTL + ED group (b) did not reach statistical significance (e). ${ }^{*} p<0.05$ when compared with CTL group; ${ }^{*} p<0.01$ when compared with CTL group; ${ }^{\#} p<0.001$ when compared with IH group; ${ }^{\# \#} p<0.01$ when compared with IH group; $\# \#$ ${ }^{\#}<0.001$ when compared with IH group. Abbreviation: $C T L$ control group; $I H$ intermittent hypoxia; $E D$ endostatin. $R T-P C R$ real-time polymerase chain reaction; $I O D$ integrated optical density 


\section{Effect of endostatin on VEGF}

The effect of endostatin on the expression of VEGF is depicted in Fig. 3. The VEGF levels in the IH group were significantly higher than that of the CTL group $(1.00 \pm 0.57$ vs. $1.61 \pm 0.50, p<0.05$ in mRNA expression levels; $360.67 \pm 14.68$ vs. $319.76 \pm 10.17, \mathrm{pg} / \mathrm{ml}, p<0.01$ in serum; $0.98 \pm 0.18$ vs. $1.29 \pm 0.31, p<0.001$ in western blotting results; $133.81 \pm 38.43$ vs. $308.27 \pm 93.41, p<0.001$ in the IHC results). Endostatin attenuated VEGF levels, and the decreased levels (no-ED treatment group - ED treatment group in normoxia or IH conditions) under IH condition were more significant than under normoxia condition: $1.04 \pm 0.41$ vs. $0.59 \pm 0.48, p<0.05$ in mRNA expression levels; $87.26 \pm 34.26$ vs. $20.95 \pm 15.99, p<0.05$ in serum; $0.59 \pm 0.22$ vs. $0.35 \pm 0.22, p<0.05$ in western blotting results. The IHC results showed that endostatin decreased the IOD staining by VEGF antibody under IH condition $(308.27 \pm 93.41$ in $\mathrm{IH}$ group vs. $91.79 \pm 41.26$ in $\mathrm{IH}+\mathrm{ED}$ group, $p<0.001)$, and similar results were failed to be observed in those under normoxia condition $(133.81 \pm 38.43$ in CTL group vs. $81.57 \pm 58.07$ in CTL + ED group, $p>0.05$ ).

\section{HE staining and MVD evaluation}

The HE staining of tumor tissues in all groups is outlined in Fig. 4. As compared to the CTL group, more necrotic tissues, nuclear deep dyeing, and nuclear division were found in IH group. Some phenomenon could be detected in ED treatment group: the decreased ratio of nuclear to the cytoplasm and the signal of 'signet-ring cells'.

Microvessel distribution is shown in Fig. 5. The MVD values were assessed by immunohistochemical staining of the CD34 antibody. Cells positive for CD34 were stained brown. Quantitative analysis revealed an increase of MVD values in $\mathrm{IH}$ group $(26.00 \pm 6.20$ vs. $17.60 \pm 4.65$ in CTL group, $p<0.001$ ); when treated with ED, MVD values both under normoxia and IH conditions were decreased $(17.60 \pm 4.65$ in CTL group vs. $11.20 \pm 2.15$ in CTL $+\mathrm{ED}$ group, $p<0.01 ; 26.00 \pm 6.20$ in IH group vs. $12.70 \pm 5.14$ in $\mathrm{IH}+\mathrm{ED}$ group, $p<0.001$ ), and the decreased levels under IH condition were more significant than that of the normoxia condition $(13.30 \pm 8.63$ vs. $6.40 \pm 5.27, p<0.05)$.

\section{Discussion}

The present study established two models of IH and tumor in mice. Micro-PET-CT was conducted to evaluate the influence of IH on tumor growth and the anti-tumor effect of endostatin under the normoxia and IH conditions. The results showed that $\mathrm{IH}$ accelerates tumor development and enhances the expression of VEGF. The micro-PET-CT results found high SUVmax values and necrotic area in the $\mathrm{IH}$-exposed mice. The anti-tumor effect of endostatin under IH condition was superior to that of normoxia condition.
CTL
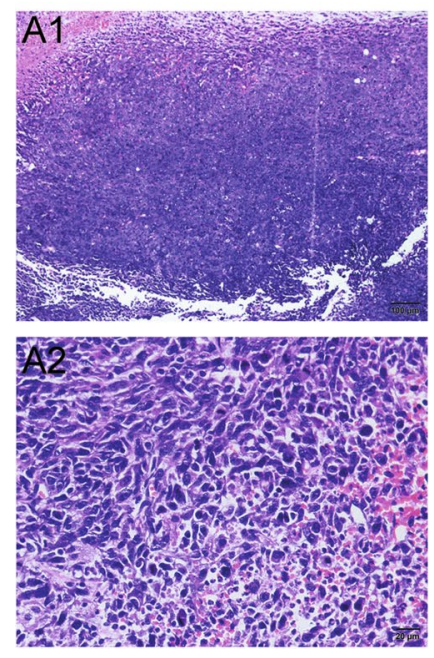
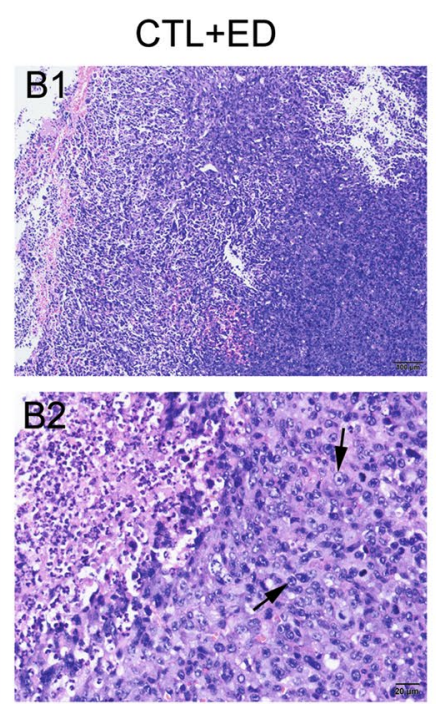

Fig. 4 HE staining of tumor tissues in all groups. Compared with the CTL group, tissue necrotic area is bigger in the IH group and endostatin therapeutic groups (CTL +ED group and $\mathrm{IH}+\mathrm{ED}$ group). As compared to mice under normoxia condition, the phenomena of nuclear deep dyeing and nuclear division (black arrows) were observed in mice that were exposed to $\mathrm{IH}$ condition (IH and IH + ED

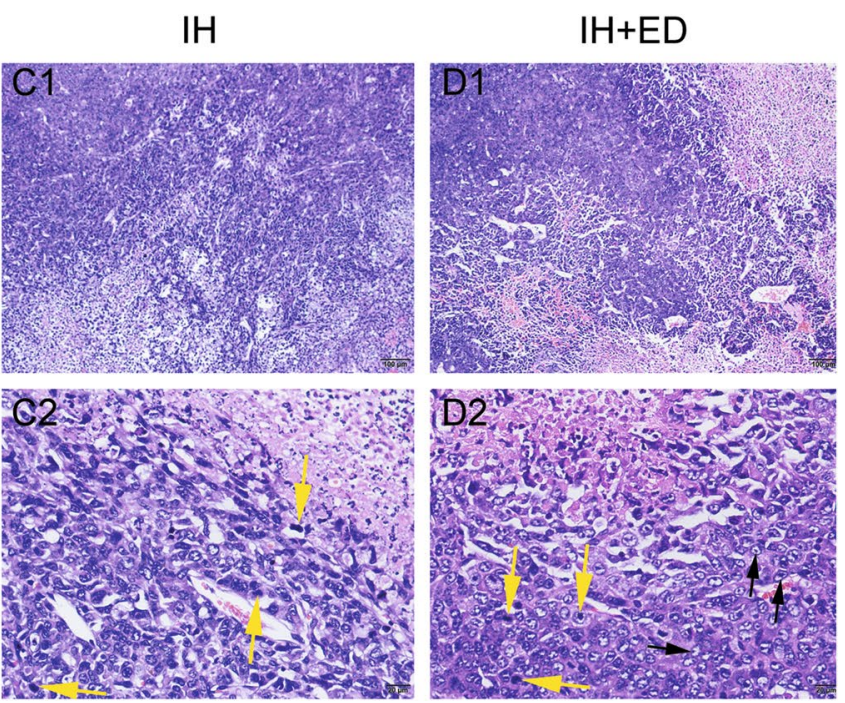

groups). In endostatin therapeutic groups (CTL + ED and IH+ED groups), the ratio of nuclear to cytoplasm was decreased, and the signal of 'signet-ring cells' (the cells rich in cytoplasm and filled with mucus, and the nucleus is squeezed on the cytoplasmic side) is presented (yellow arrows) 

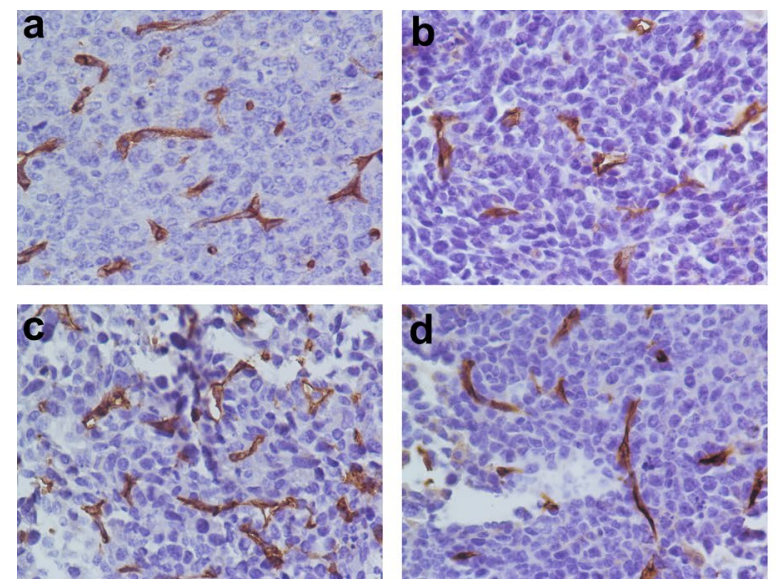

Fig. 5 Microvessel density was evaluated by immunohistochemical staining of CD34. a CTL group; b CTL + ED group; c IH group; d $\mathrm{IH}+\mathrm{ED}$ group. As compared to mice in the CTL group, those in the IH group had high MVD values. The MVD values could be lowed after treating with endostatin, and the treated effect was more obvi-

IH is a novel pathophysiological hallmark in OSA patients. Increasing attention has been paid to illustrating the exact relationship between $\mathrm{IH}$ and tumor $[11,12]$. Almendros et al. [13] and Yoon et al. [31] demonstrated that IH facilitates tumor growth. Meanwhile, the previous studies both in vivo and in vitro also prove that IH induces migration and metastasis in different types of tumor [15-18]. Our results were consistent with previous studies. The tumor weight and volume were greater in mice exposed to IH than those exposed to normoxia condition. VEGF levels in both serum and tumor tissue were increased after IH exposure, which was consistent with the previous study [32]. The results of the present study indicated that OSA-liked IH can aggravate tumor growth, facilitating the angiogenesis in tumor tissue. The connection between IH and tumor progression was also confirmed by clinical investigation. Several studies exhibited high prevalence and mortality of cancer in OSA patients. Further analysis found that the polysomnographic parameters, such as apnea-hypopnea index and oxygen desaturation index, were closely associated with cancer incidence and mortality [3, 5-7].

Robust evidence elucidated that endostatin can inhibit endothelial proliferation and angiogenesis in tumor [33, 34]. Few studies addressed the therapeutic effect of endostatin under IH condition. The results of the present study confirmed that endostatin can slow down tumor development. More tumor necrotic area can be found after endostatin treatment. The MVD and VEGF levels were reduced by endostatin. Furthermore, the anti-tumor effect is more significant when tumor-bearing mice is subjected to the IH condition mimicking OSA. The findings of the present study were similar to several experimental and clinical studies. Zhu et al. [21], worked on the effect of endostatin on esophageal

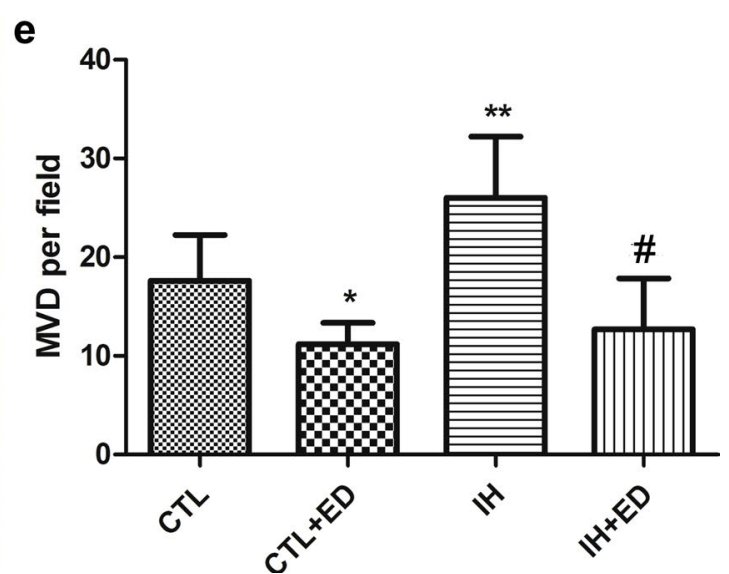

ous in mice exposed to IH condition. $* p<0.05$ when compared with CTL group; $* * p<0.01$ when compared with CTL group; ${ }^{\#} p<0.001$ when compared with IH group (e). Abbreviations: MVD microvessel density; $C T L$ control group; $E D$ endostatin; $I H$ intermittent hypoxia

squamous cell cancer in mice and found that endostatin can suppress tumor growth, reducing MVD, and inhibiting the expression of VEGF in tumor tissue. These effects were more significant when endostatin combines with radiation therapy. The anti-cancer effect of endostatin possibly attributes to its normalized vasculature and reduced hypoxia [21]. Another study [35] indicated that endostatin plus chemotherapy leads to a more potent inhibition of tumor growth. In human research, a randomized controlled trial from a research group in China found that the combination of vinorelbine-cisplatin with endostatin can increase therapeutic response in non-small cell lung cancer and bone metastasis patients [20]. Two meta-analysis studies [19, 36] elicited that when compared to platinum-based chemotherapy, a combination of endostatin can improve chemotherapeutic response rate, disease control rate, and the time to progress, without increasing the incidence of adverse events. No data were found regarding the anti-tumor effect of endostatin among OSA population before; therefore, further investigation is required.

Compared with conservative CT scan, PET-CT is an imaging technique that allows for the non-invasive visualization and quantification of metabolic processes in vivo. PET using ${ }^{18} \mathrm{~F}$-FDG shows the increased glycolytic rate of malignant cells in tumors [37]. PET-CT can be used to evaluate tumor metabolic processes and treatment response [38]. In the present study, we applied micro-PET-CT, in which ${ }^{18} \mathrm{~F}$-FDG was used as a tracer, to assess the IH-exposed tumor features in live animals. High SUVmax values were observed in the tumor after IH exposure, while endostatin treatment can attenuate the SUVmax values and increase tumor necrotic area both under normoxia and IH conditions. The micro-PET-CT 
information from our study illustrated that increased tumor metabolic levels were observed in the IH condition. The reduced levels of tumor metabolism treated by endostatin were more obvious in the IH condition than in normoxia condition. Further clinical studies are warranted to figure out whether PET-CT can be conducted to evaluate the tumor metabolic levels in OSA patients.

The present study has its strength. Principally, it was the first study to determine the anti-tumor effect of endostatin in a mouse model mimicking OSA. Furthermore, the present study is the first to use micro-PET-CT to access the influence of IH on tumor and find out the anti-tumor effect of endostatin in live animals.

However, the present study has several limitations. First, when applying PET-CT to evaluate the anti-tumor effect of endostatin, we used ${ }^{18} \mathrm{~F}$-FDG as PET-CT tracer, instead of those probes specific to epidermal growth factor receptor (EGFR), such as ${ }^{18} \mathrm{~F}-\mathrm{FBEM}-\mathrm{Cys}-\mathrm{Z}_{\mathrm{EGFR}: 1907}$ and ${ }^{64} \mathrm{Cu}$-cetuximab-F $\left(\mathrm{ab}^{\prime}\right)_{2}$. The specificity of ${ }^{18} \mathrm{~F}$-FDG is possibly inferior to those EGFR probes. Additional study using specific EGFR probes as PET-CT tracers might be warranted to evaluate the anti-tumor effect of endostatin in an OSA mouse model. Second, only on-site tumor metabolic behavior was observed via micro-PET-CT, the metastatic lesion was not evaluated as the previous study $[15,16,25]$; therefore, further study is required. Third, we only judge the anti-tumor effect of endostatin alone under IH condition. Further study should be focused on the combined effects of endostatin plus chemotherapy or radiotherapy in OSA-mimicking animal or patients. Finally, as previous studies showed [14-16], the IH exposure time was relatively short in our current study (5 weeks); ongoing study is required to figure out the effect of long-term IH exposure on tumor mice.

\section{Conclusions}

The present study demonstrates that $\mathrm{IH}$ can aggravate tumor progress in a mouse model of sleep apnea, and the antitumor effect of endostatin under IH condition appears to be superior to that of normoxia condition.

Author contributions $\mathrm{X}-\mathrm{B}, \mathrm{Z}, \mathrm{Y}, \mathrm{Z}$, and $\mathrm{H}-\mathrm{Q}, \mathrm{Z}$ : conception and design. B-B, F, Y-Y, Y, Y-T, L, Y, W and Y-P, D: collection and assembly of data. X-B, Z, Y-Y, Y, L-D, C, X, L, C-Y, K, and H-Q, Z: data analysis and interpretation. Manuscript writing: all authors. Final approval of the manuscript: all authors.

Funding This work was supported by Grant $3502 Z 20154019$ for Fund from Xiamen Science and Technology Bureau, Grant 2018-2-65 for Youth Research Fund from Fujian Provincial Health Bureau, and Grant 2018J01393 for Fund from Natural Science Foundation of Fujian Province, China.

\section{Compliance with ethical standards}

Conflict of interest The authors declare that they have no conflict of interest.

Ethical approval The study was approved by the Ethics Commi ttee of Zhongshan Hospital, Xiamen University.

Informed consent The research did not involve human participants.

Availability of data and material All data generated or analyzed during this study are included in this published article.

Open Access This article is distributed under the terms of the Creative Commons Attribution 4.0 International License (http://creativeco mmons.org/licenses/by/4.0/), which permits unrestricted use, distribution, and reproduction in any medium, provided you give appropriate credit to the original author(s) and the source, provide a link to the Creative Commons license, and indicate if changes were made.

\section{References}

1. Jordan AS, McSharry DG, Malhotra A. Adult obstructive sleep apnoea. The Lancet. 2014;383(9918):736-47. https://doi. org/10.1016/S0140-6736(13)60734-5.

2. Peker Y, Hedner J, Norum J, Kraiczi H, Carlson J. Increased incidence of cardiovascular disease in middle-aged men with obstructive sleep apnea: a 7-year follow-up. Am J Respir Crit Care Med. 2002;166(2):159-65. https://doi.org/10.1164/rccm.2105124.

3. Chen JC, Hwang JH. Sleep apnea increased incidence of primary central nervous system cancers: a nationwide cohort study. Sleep Med. 2014;15(7):749-54.

4. Martinez-Garcia MA, Campos-Rodriguez F, Duran-Cantolla J, de la Pena M, Masdeu MJ, Gonzalez M, et al. Obstructive sleep apnea is associated with cancer mortality in younger patients. Sleep Med. 2014;15(7):742-8. https://doi.org/10.1016/j.sleep .2014.01.020.

5. Campos-Rodriguez F, Martinez-Garcia MA, Martinez M, DuranCantolla J, de la Pena M, Masdeu MJ, et al. Association between obstructive sleep apnea and cancer incidence in a large multicenter Spanish cohort. Am J Respir Crit Care Med. 2013;187(1):99-105.

6. Marshall NS, Wong KK, Cullen SR, Knuiman MW, Grunstein RR. Sleep apnea and 20-year follow-up for all-cause mortality, stroke, and cancer incidence and mortality in the Busselton health study cohort. J Clin Sleep Med. 2014;10(4):355-62. https://doi. org/10.5664/jcsm.3600.

7. Nieto FJ, Peppard PE, Young T, Finn L, Hla KM, Farre R. Sleep-disordered breathing and cancer mortality results from the Wisconsin Sleep Cohort Study. Am J Respir Crit Care Med. 2012;186(2):190-4.

8. Dreher M, Kruger S, Schulze-Olden S, Keszei A, Storre JH, Woehrle H, et al. Sleep-disordered breathing in patients with newly diagnosed lung cancer. BMC Pulm Med. 2018;18(1):72. https://doi.org/10.1186/s12890-018-0645-1.

9. Payne RJ, Hier MP, Kost KM, Black MJ, Zeitouni AG, Frenkiel S, et al. High prevalence of obstructive sleep apnea among patients with head and neck cancer. J Otolaryngol. 2005;34(5):304-11.

10. Li L, Lu J, Xue W, Wang L, Zhai Y, Fan Z, et al. Target of obstructive sleep apnea syndrome merge lung cancer: based on big data platform. Oncotarget. 2017;8(13):21567-78. https://doi. org/10.18632/oncotarget.15372. 
11. Almendros I, Gozal D. Intermittent hypoxia and cancer: undesirable bed partners? Respir Physiol Neurobiol. 2017. https://doi. org/10.1016/j.resp.2017.08.008.

12. Michiels $\mathrm{C}$, Tellier $\mathrm{C}$, Feron O. Cycling hypoxia: a key feature of the tumor microenvironment. Biochim Biophys Acta. 2016;1866(1):76-86. https://doi.org/10.1016/j.bbcan .2016.06.004.

13. Almendros I, Montserrat JM, Ramirez J, Torres M, Duran-Cantolla J, Navajas D, et al. Intermittent hypoxia enhances cancer progression in a mouse model of sleep apnoea. Eur Respir J. 2012;39(1):215-7. https://doi.org/10.1183/09031936.00185110.

14. Yoon DW, So D, Min S, Kim J, Lee M, Khalmuratova R, et al. Accelerated tumor growth under intermittent hypoxia is associated with hypoxia-inducible factor-1-dependent adaptive responses to hypoxia. Oncotarget. 2017;8(37):61592-603. https://doi. org/10.18632/oncotarget.18644.

15. Li L, Ren F, Qi C, Xu L, Fang Y, Liang M, et al. Intermittent hypoxia promotes melanoma lung metastasis via oxidative stress and inflammation responses in a mouse model of obstructive sleep apnea. Respir Res. 2018;19(1):28. https://doi.org/10.1186/s1293 1-018-0727-x.

16. Chen A, Sceneay J, Godde N, Kinwel T, Ham S, Thompson $\mathrm{EW}$, et al. Intermittent hypoxia induces a metastatic phenotype in breast cancer. Oncogene. 2018;37(31):4214-25. https://doi. org/10.1038/s41388-018-0259-3.

17. Liu L, Liu W, Wang L, Zhu T, Zhong J, Xie N. Hypoxia-inducible factor 1 mediates intermittent hypoxia-induced migration of human breast cancer MDA-MB-231 cells. Oncol Lett. 2017;14(6):7715-22. https://doi.org/10.3892/ol.2017.7223.

18. Gutsche K, Randi EB, Blank V, Fink D, Wenger RH, Leo C, et al. Intermittent hypoxia confers pro-metastatic gene expression selectively through NF-kappaB in inflammatory breast cancer cells. Free Radic Biol Med. 2016;101:129-42. https://doi.org/10.1016/j. freeradbiomed.2016.10.002.

19. An J, Lv W. Endostar (rh-endostatin) versus placebo in combination with vinorelbine plus cisplatin chemotherapy regimen in treatment of advanced non-small cell lung cancer: a meta-analysis. Thorac Cancer. 2018;9(5):606-12. https://doi.org/10.1111/17597714.12626

20. Zhang R, Wang ZY, Li YH, Lu YH, Wang S, Yu WX, et al. Dynamic contrast-enhanced MRI to predict response to vinorelbine-cisplatin alone or with rh-endostatin in patients with nonsmall cell lung cancer and bone metastases: a randomised, doubleblind, placebo-controlled trial. Lancet. 2016;388(Suppl 1):S95. https://doi.org/10.1016/S0140-6736(16)32022-0.

21. Zhu H, Yang X, Ding Y, Liu J, Lu J, Zhan L, et al. Recombinant human endostatin enhances the radioresponse in esophageal squamous cell carcinoma by normalizing tumor vasculature and reducing hypoxia. Sci Rep. 2015;5:14503. https://doi.org/10.1038/ srep14503.

22. Sun J, Deng L, Duan Y, Chen F, Wang X, Li D, et al. Inhibitory effect of endostatin combined with paclitaxel-cisplatin on breast cancer in xenograft-bearing mice. Exp Ther Med. 2012;3(2):159 64. https://doi.org/10.3892/etm.2011.384.

23. Vilaseca A, Campillo N, Torres M, Musquera M, Gozal D, Montserrat JM, et al. Intermittent hypoxia increases kidney tumor vascularization in a murine model of sleep apnea. PLoS One. 2017;12(6):e0179444. https://doi.org/10.1371/journal.pone.01794 44.

24. Toffoli S, Roegiers A, Feron O, Van Steenbrugge M, Ninane N, Raes M, et al. Intermittent hypoxia is an angiogenic inducer for endothelial cells: role of HIF-1. Angiogenesis. 2009;12(1):47-67. https://doi.org/10.1007/s10456-009-9131-y.
25. Almendros I, Montserrat JM, Torres M, Dalmases M, Cabanas ML, Campos-Rodriguez F, et al. Intermittent hypoxia increases melanoma metastasis to the lung in a mouse model of sleep apnea. Respir Physiol Neurobiol. 2013;186(3):303-7. https://doi. org/10.1016/j.resp.2013.03.001.

26. Almendros I, Wang Y, Becker L, Lennon FE, Zheng J, Coats BR, et al. Intermittent hypoxia-induced changes in tumor-associated macrophages and tumor malignancy in a mouse model of sleep apnea. Am J Respir Crit Care Med. 2014;189(5):593-601. https ://doi.org/10.1164/rccm.201310-1830OC.

27. National Research Concil (US). Committee for the update of the guide for the care and use of laboratory animals. Guide for the care and use of laboratory animals. 8th ed. Washington DC: National Academies Press; 2011.

28. Chen XY, Zeng YM, Zhang YX, Wang WY, Wu RH. Effect of chronic intermittent hypoxia on theophylline metabolism in mouse liver. Chin Med J (Engl). 2013;126(1):118-23.

29. Su X, Cheng K, Liu Y, Hu X, Meng S, Cheng Z. PET imaging of insulin-like growth factor type 1 receptor expression with a $64 \mathrm{Cu}-$ labeled Affibody molecule. Amino Acids. 2015;47(7):1409-19. https://doi.org/10.1007/s00726-015-1975-4.

30. Weidner N, Semple JP, Welch WR, Folkman J. Tumor angiogenesis and metastasis - correlation in invasive breast carcinoma. N Engl J Med. 1991;324(1):1-8. https://doi.org/10.1056/NEJM1 99101033240101.

31. Yoon DW, So D, Min S, Kim J, Lee M, Khalmuratova R, et al. Accelerated tumor growth under intermittent hypoxia is associated with hypoxia-inducible factor-1-dependent adaptive responses to hypoxia. Oncotarget. 2017. https://doi.org/10.18632/oncotarget .18644.

32. Almendros I, Montserrat JM, Torres M, Bonsignore MR, Chimenti L, Navajas D, et al. Obesity and intermittent hypoxia increase tumor growth in a mouse model of sleep apnea. Sleep Med. 2012;13(10):1254-60. https://doi.org/10.1016/j.sleep .2012.08.012.

33. Folkman J. Antiangiogenesis in cancer therapy-endostatin and its mechanisms of action. Exp Cell Res. 2006;312(5):594-607. https://doi.org/10.1016/j.yexcr.2005.11.015.

34. O'Reilly MS, Boehm T, Shing Y, Fukai N, Vasios G, Lane WS, et al. Endostatin: an endogenous inhibitor of angiogenesis and tumor growth. Cell. 1997;88(2):277-85.

35. Zhu LP, Xing J, Wang QX, Kou L, Li C, Hu B, et al. Therapeutic efficacy of recombinant human endostatin combined with chemotherapeutics in mice-transplanted tumors. Eur J Pharmacol. 2009;617(1-3):23-7. https://doi.org/10.1016/j.ejpha r.2009.07.003.

36. Rong B, Yang S, Li W, Zhang W, Ming Z. Systematic review and meta-analysis of Endostar (rh-endostatin) combined with chemotherapy versus chemotherapy alone for treating advanced nonsmall cell lung cancer. World J Surg Oncol. 2012;10:170. https:// doi.org/10.1186/1477-7819-10-170.

37. Fletcher JW, Djulbegovic B, Soares HP, Siegel BA, Lowe VJ, Lyman GH, et al. Recommendations on the use of 18F-FDG PET in oncology. J Nucl Med. 2008;49(3):480-508. https://doi. org/10.2967/jnumed.107.047787.

38. Walter MA, Hildebrandt IJ, Hacke K, Kesner AL, Kelly O, Lawson GW, et al. Small-animal PET/CT for monitoring the development and response to chemotherapy of thymic lymphoma in Trp53-/- mice. J Nucl Med. 2010;51(8):1285-92. https://doi. org/10.2967/jnumed.109.073585. 


\section{Affiliations}

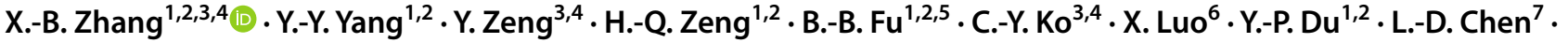
Y.-T. Lai ${ }^{1,2} \cdot$ Y. Wu ${ }^{1,2}$

X.-B. Zhang

zhangxiaobincn@xmu.edu.cn

Y.-Y. Yang

913240424@qq.com

B.-B. Fu

414916378@qq.com

C.-Y. Ko

yuanmomoko@gmail.com

X. Luo

lurowan@aliyun.com

Y.-P. Du

duyanping@21cn.com

L.-D. Chen

361328175@qq.com

Y.-T. Lai

441564335@qq.com

$\mathrm{Y} . \mathrm{Wu}$

1357100395@qq.com
Department of Respiratory Medicine, Zhongshan Hospital, Xiamen University, Xiamen, Fujian, China

2 Teaching Hospital of Fujian Medical University, Siming District, Xiamen, Fujian, China

3 Second Clinical Medical College of Fujian Medical University, Fuzhou, Fujian, China

4 Department of Pulmonary and Critical Care Medicine, Center of Respiratory Medicine of Fujian Province, The Second Affiliated Hospital of Fujian Medical University, Quanzhou, China

5 Medical College of Xiamen University, Xiamen 361005, China

6 Department of Computer Science, Xiamen University, Xiamen 361005, China

7 Department of Respiratory Medicine, Zhangzhou Affiliated Hospital of Fujian Medical University, Xiangcheng District, Zhangzhou, China 